\title{
Effects of pilocarpine on the morphology of the human outflow apparatus
}

\author{
IAN GRIERSON, WILliaM R. LEE, AND SHAHIDA ABRAHAM \\ From the University Department of Ophthalmology, University of Glasgow
}

SUMMARY The morphology of the outflow apparatus in human eyes which had been treated topically with either 2 or $4 \%$ pilocarpine on 4 occasions at 6-hourly intervals before enucleation was compared with an untreated control series of eyes from patients in a similar age range. In the pilocarpinetreated group the scleral spur was pulled posteriorly and internally, so that the angle formed by the scleral spur and the scleral sulcus was significantly greater in the treated than the untreated series. The change in attitude of the scleral spur produced widening of the spaces between the corneoscleral trabeculae and distension of the endothelial meshwork. It was shown from counts of giant vacuoles in the endothelium of Schlemm's canal that the incidence of giant vacuoles in the pilocarpine-treated group was greater than twice that in the controls. It would appear that pilocarpine produces alterations in the configuration of the outflow apparatus which would promote the drainage of aqueous humour.

Pilocarpine is widely used in ophthalmic practice to decrease the resistance to aqueous outflow which occurs in primary and secondary open-angle glaucoma (Duke-Elder, 1958). Thus, the material which is made available for pathological study by enucleation or by the trabeculectomy procedure has almost invariably been subjected to pilocarpine treatment. The interpretation of the changes observed in pathological specimens is to some extent handicapped by the lack of documentation of the effect of pilocarpine on the morphology of the normal human outflow system, although recent work has demonstrated an increase in the facility of aqueous outflow in the normal human eye (Gaasterland et al., 1975; Mapstone, 1977), and morphological changes should therefore be expected.

The use of experimental animals in physiological and morphological studies on the effect of pilocarpine on the outflow system has revealed important species variations (Bárány, 1966). However, it is generally accepted that the principal action of the drug is through contraction of the ciliary muscle, which in turn displaces the scleral spur and the trabecular tissues, so that spaces for the drainage of aqueous humour are widened (Duke-Elder, 1958). This alteration in morphology has been demonstrated by

Address for reprints: Dr W. R. Lee, University of Glasgow, Department of Ophthalmology, 38 Church Street, Glasgow G11 6NT light microscopy in the rhesus monkey by Flocks and Zweng (1957). In addition the electron microscopic studies of Lütjen-Drecoll (1973) using stumptail monkeys have correlated an increase in physiological facility measurements with an increase in the area of electron-lucent spaces in the outer layers of the meshwork.

In order to establish that morphological changes occur in the human eye after pilocarpine the outflow system in a group of eyes which were previously treated with the drug was compared with that in a control group. The investigation was directed towards a demonstration of $(a)$ displacement effects on the scleral spur and $(b)$ changes in the spatial relationships between the various cellular components of the trabecular meshwork. A quantitative analysis was performed by means of an established technique (Grierson and Lee, 1977) by light microscopy, on the giant vacuoles in the lining endothelium of Schlemm's canal. These vacuoles have been described in the normal lining endothelium and have been implicated in the transfer of aqueous humour (Holmberg, 1965; Kayes, 1967; Tripathi, 1968, 1972, 1974; Inomata et al., 1972). It has been shown from experimental studies that giant vacuoles (Johnstone and Grant, 1973; Grierson and Lee, 1974, 1977) and transendothelial channels (Lee and Grierson, 1974; Grierson and Lee, 1975a; Svedbergh, 1976) are temporary pressure-sensitive structures. On this basis it could be expected that 
they would show either qualitative or quantitative alterations in response to drug-induced changes in aqueous outflow.

\section{Materials and methods}

Nineteen human eyes were available for the investigation. The eyes were enucleated in treatment of small malignant melanomata in which there was no clinical or histopathological evidence of abnormality in the tissues of the anterior segment.

In 8 patients the eyes to be enucleated were treated on 4 occasions (at 6-hourly intervals) with 2 to $4 \%$ pilocarpine eye drops before enucleation The remaining 11 eyes were untreated and served as a control series. Clinical outflow facility measurements were not determined because the material was submitted from a variety of sources in the west of Scotland. The details of the age and sex of the patients are listed in Table 1. There was no significant difference between the age ranges of the 2 groups.

After a routine surgical enucleation the eyes were immersed in 2 to $4 \%$ glutaraldehyde buffered with $0 \cdot 1 \mathrm{M}$ Sorensen's phosphate buffer. Subsequently meridional blocks of limbal tissue were removed from the calottes which were not required for diagnostic pathology. The blocks were buffer washed, post-fixed in $1 \%$ osmium tetroxide, rewashed in buffer, and dehydrated through graded alcohols. The tissue was cleared in propylene oxide and embedded in Araldite.

Thick $(1$ to $2 \mu \mathrm{m})$ and thin 600 to $800 \AA$ (60 to $80 \mathrm{~nm}$ )) sections were cut on an LKB Ultrotome III. The thick sections were stained with toluidine blue and the thin sections were double stained with uranyl acetate and lead citrate. The transmission electron microscope which was used for the investigation was a Phillips 301 .

The toluidine blue stained sections were used for
Table 1 The age and sex of individuals in the pilocarpine-treated and the untreated group and the values obtained for the photomicrographic analysis of the angle subtended by the scleral spur and the scleral sulcus. For each eye a mean value ( $\pm S D$ ) was derived from 10 blocks. By Student's t-test the difference between the groups was highly significant $(P<0.001)$

\begin{tabular}{|c|c|c|c|c|c|}
\hline & $\begin{array}{l}\text { Treated } \\
\text { Age/Sex }\end{array}$ & $\begin{array}{l}\text { Angle subtended } \\
\text { by scleral spur } \\
\text { and scleral } \\
\text { sulcus (degrees) }\end{array}$ & & $\begin{array}{l}\text { Control } \\
\text { Age/Sex }\end{array}$ & $\begin{array}{l}\text { Angle subtended } \\
\text { by scleral spur } \\
\text { and scleral } \\
\text { sulcus (degrees) }\end{array}$ \\
\hline 1. & $29 \mathrm{~F}$ & $34 \cdot 4 \pm 7 \cdot 4$ & 1. & $21 \mathrm{~F}$ & $13.8 \pm 5.4$ \\
\hline 2. & $46 \mathrm{M}$ & $26 \cdot 1 \pm 5 \cdot 2$ & 2. & $31 \mathrm{~F}$ & $18 \cdot 5 \pm 6 \cdot 5$ \\
\hline 3. & $46 \mathrm{~F}$ & $30 \cdot 5 \pm 4 \cdot 3$ & 3. & $38 \mathrm{~F}$ & $14 \cdot 1 \pm 11 \cdot 0$ \\
\hline 4. & $50 \mathrm{~F}$ & $38 \cdot 3 \pm 6 \cdot 3$ & 4. & $53 \mathrm{M}$ & $18.9 \pm 5.0$ \\
\hline 5. & $55 \mathrm{~F}$ & $35 \cdot 0 \pm 7 \cdot 1$ & 5. & $54 \mathrm{M}$ & $8 \cdot 8 \pm 3 \cdot 7$ \\
\hline 6. & $60 \mathrm{~F}$ & $41 \cdot 0 \pm 6 \cdot 6$ & 6. & $58 \mathrm{M}$ & $17 \cdot 9 \pm 5 \cdot 3$ \\
\hline 7. & $61 \mathrm{~F}$ & $30.6 \pm 6.6$ & 7. & $63 \mathrm{M}$ & $18 \cdot 2 \pm 6 \cdot 6$ \\
\hline \multirow[t]{4}{*}{8.} & $75 \mathrm{M}$ & $28 \cdot 6 \pm 6 \cdot 2$ & 8. & $63 \mathrm{~F}$ & $21 \cdot 8 \pm 7 \cdot 0$ \\
\hline & \multirow[t]{3}{*}{$\begin{array}{l}53 \pm 14 \\
(\text { Mean }) \\
( \pm \text { SD) }\end{array}$} & $\begin{array}{l}33 \cdot 1 \pm 5 \cdot 0 \\
\text { (Grand mean) } \\
( \pm \text { SD) }\end{array}$ & $\begin{array}{l}9 . \\
10 .\end{array}$ & $\begin{array}{l}68 \mathrm{~F} \\
75 \mathrm{~F}\end{array}$ & $\begin{array}{l}24.6 \pm 5.8 \\
18.9 \pm 3.9\end{array}$ \\
\hline & & & 11. & $80 \mathrm{M}$ & $9 \cdot 6 \pm 4.8$ \\
\hline & & & & $\begin{array}{l}55 \pm 15 \\
\text { (Mean) } \\
( \pm \text { SD) }\end{array}$ & $\begin{array}{l}16 \cdot 8 \pm 4.8 \\
\text { (Grand mean) } \\
( \pm \text { SD) }\end{array}$ \\
\hline
\end{tabular}

the quantitative light microscopic studies. For the assessment of scleral spur displacement 1 thick section was selected for photomicrography from a group taken from each of 10 tissue blocks from each eye. On each photomicrograph a measurement was made of the angle subtended by the axis of the scleral spur on a line which passed through the anterior and posterior limits of the scleral sulcus (Fig. 1).
Fig. 1 A photomicrograph to demonstrate the technique for measurent of the angle subtended by the scleral spur on the scleral sulcus in the eye of a 21-year-old female (Toluidine blue, $\times 270$ )

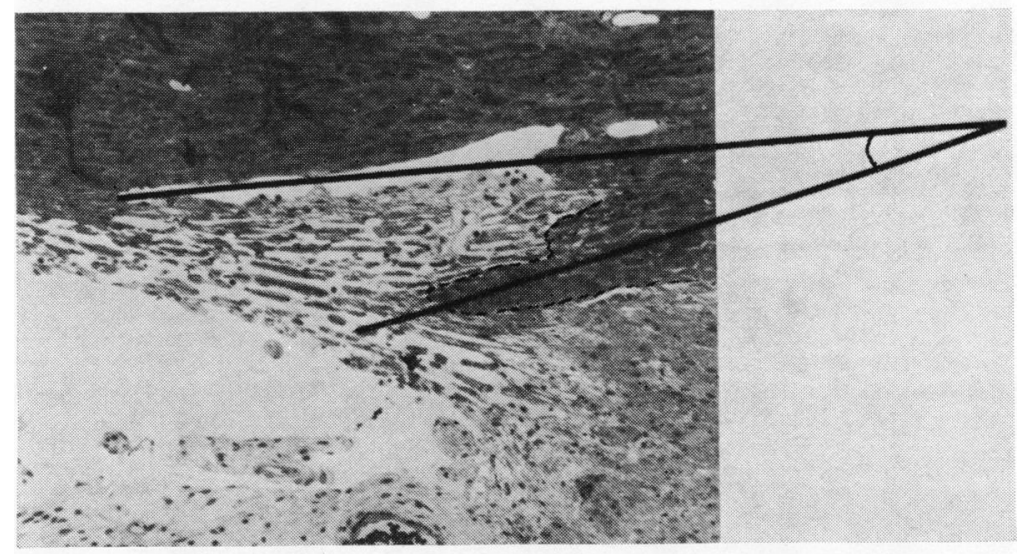


To determine the incidence of giant vacuoles in the lining endothelium of Schlemm's canal these structures were counted by means of an oil immersion lens on 1 thick section from each of 10 tissue blocks from each eye. This technique has been reported previously (Grierson and Lee, 1977).

\section{Results}

There was considerable variation in the general appearance of the outflow apparatus in the 2 groups. However, several features distinguished the outflow apparatus of the pilocarpine-treated series from the control group.

Fig. 2 Photomicrographs of the angular tissues in (a) $a$ 68-year-old female untreated eye, and (b) a 50-year-old female treated eye. Note the internal displacement of the scleral spur and the distension of the outer meshwork in the pilocarpine-treated eye (Toluidine blue, $\times 260$ )
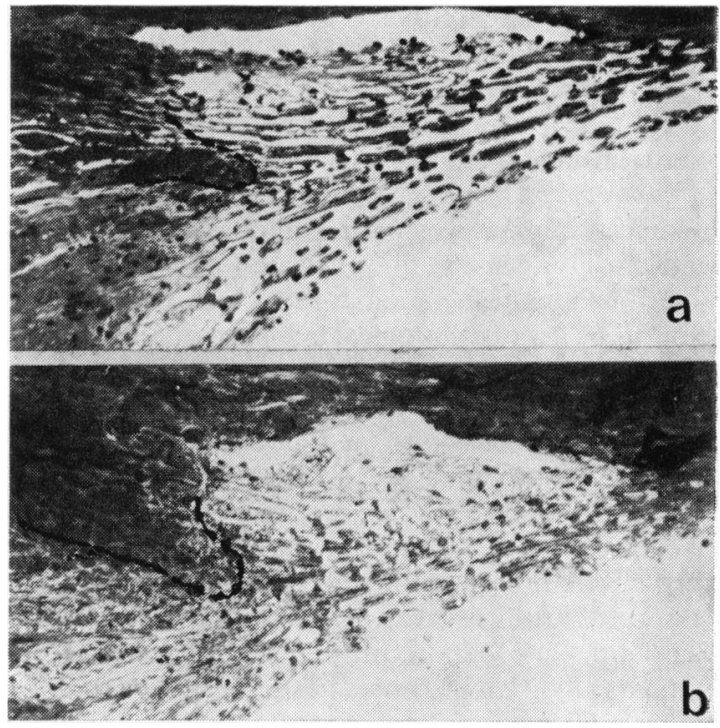

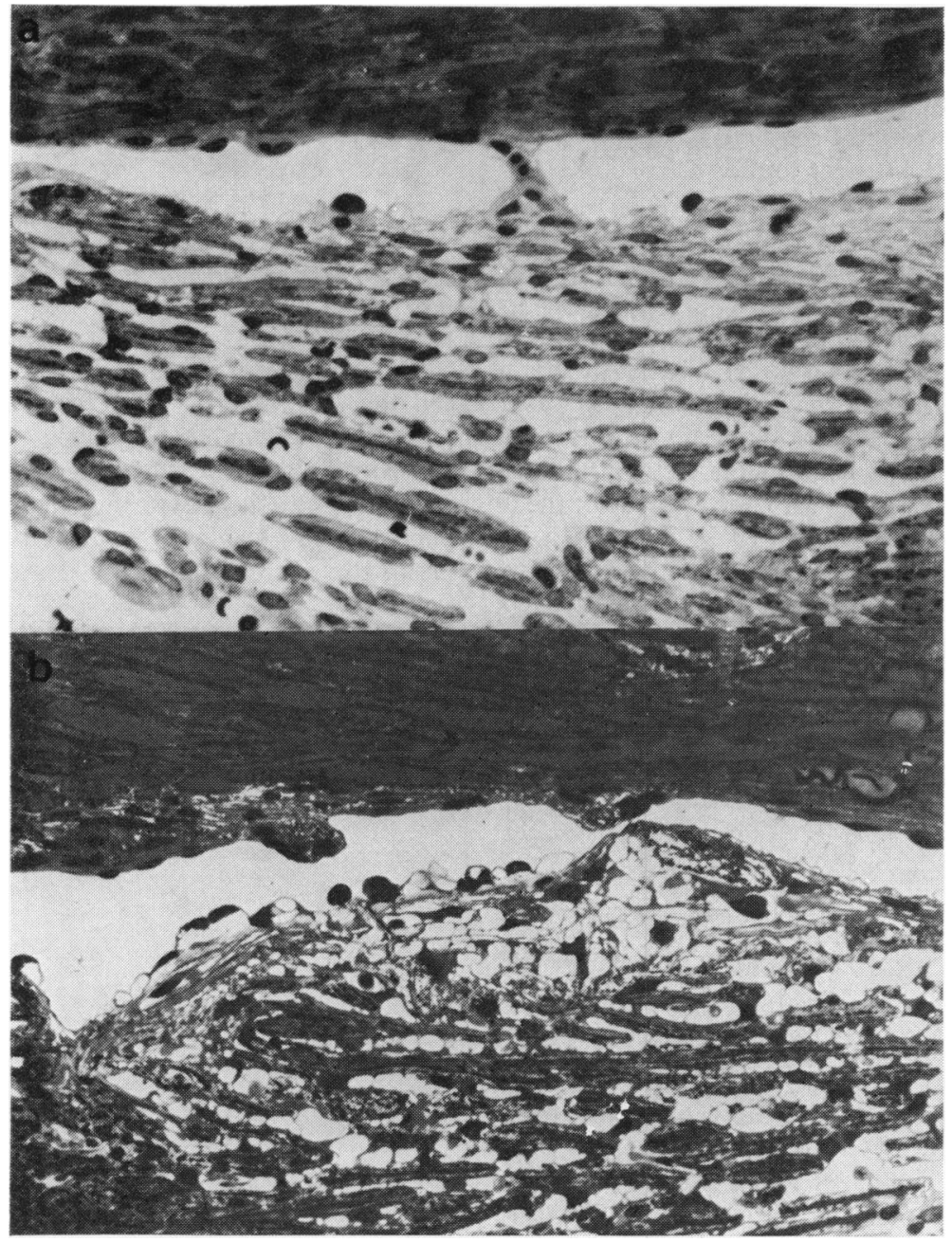

Fig. 3 Photomicrographs to illustrate the comparison between the outer (endothelial) meshwork in (a) a 21-year-old female untreated eye, and (b) a 60-yearold female pilocarpine-treated eye. In (b) note the distension of the endothelial meshwork and the increased frequency of giant vacuoles. (Toluidine blue, $\times 610$ ) 


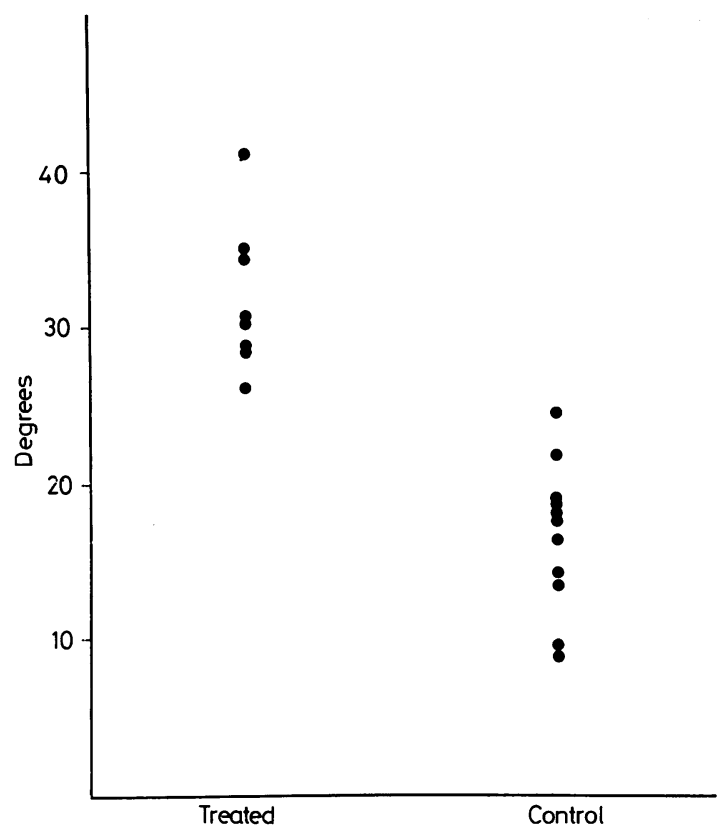

Fig. 4 Graph to show the mean values in degrees obtained for the angle subtended by the scleral spur on the scleral sulcus in the treated and the control group

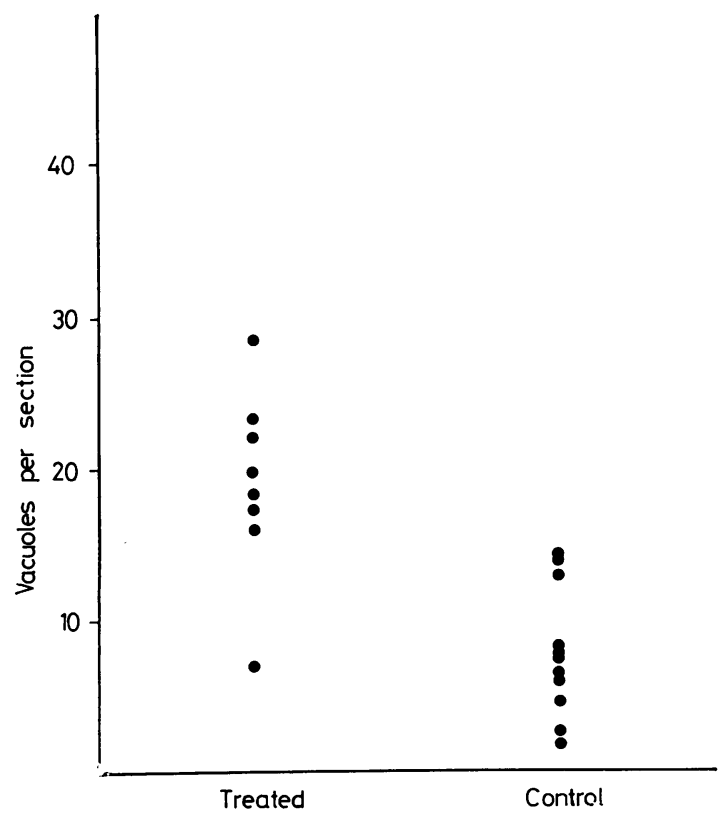

Fig. 5 Graph to show the mean values obtained for vacuoles per section in the treated and the control group

\section{LIGHT MICROSCOPY}

The impression gained from meridional sections through the outflow apparatus was that the scleral spur was pulled both internally and into a more posterior position (Fig. 2). The outer meshwork was more distended in the drug-treated than in the untreated drainage system, so that there were widely dilated spaces between the network of cells in the endothelial meshwork (Figs. 2 and 3). Dilatation of the outer meshwork narrowed the lumen of Schlemm's canal. In addition it appeared that in

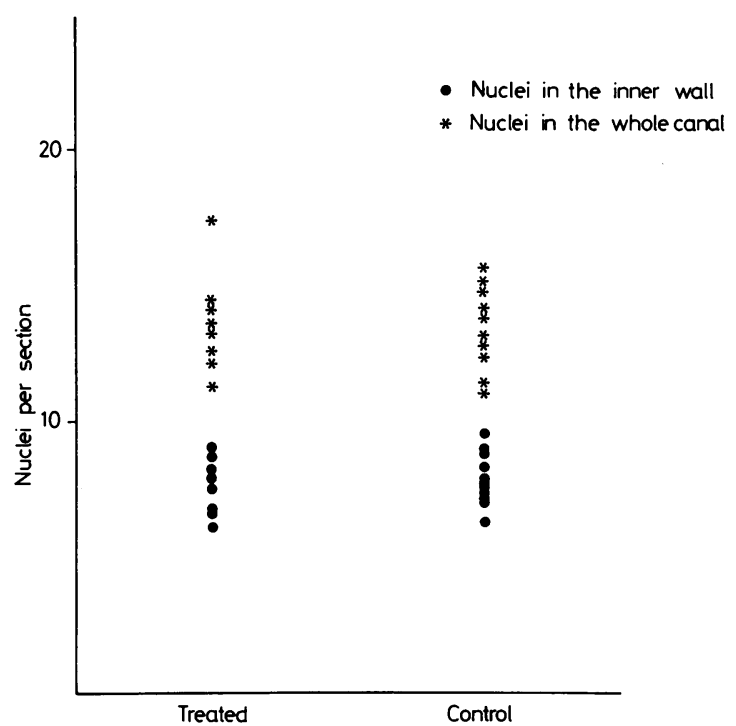

Fig. 6 Graph to show the mean values for nuclei in the endothelium lining Schlemm's canal in the treated and control group

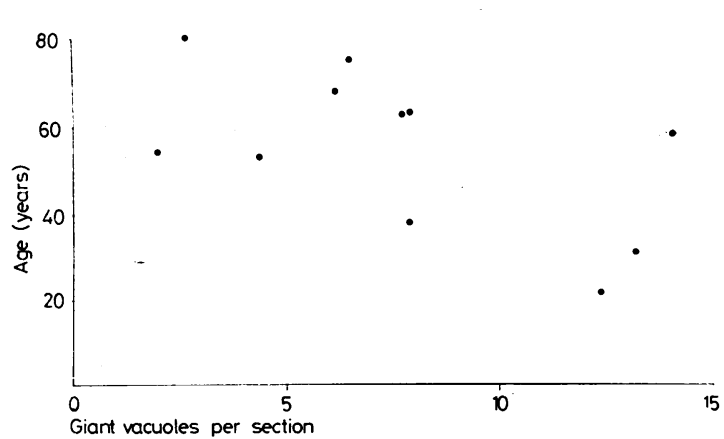

Fig. 7 Graph to show the plot of age against the mean value for giant vacuoles per section in each eye in the control group 
Table 2 The values obtained by a quantitative light microscopic analysis of the numbers of nuclei and giant vacuoles in the endothelium of Schlemm's canal. The mean $( \pm S D)$ for each eye was derived from 1 section from each of 10 blocks. Levels of significance (Student t-test) between grand means indicated by symbols $(\dagger P<0 \cdot 1),(\ddagger P<0 \cdot 001)$

\begin{tabular}{|c|c|c|c|c|c|}
\hline & \multicolumn{2}{|c|}{ Nuclei in endothelium of Schlemm's canal } & \multicolumn{3}{|c|}{ Giant vacuoles in endothelium of Schlemm's canal } \\
\hline & Inner wall & Total & Inner wall & Remainder & Total \\
\hline \multicolumn{6}{|l|}{ Treated } \\
\hline 1. $29 \mathrm{~F}$ & $8 \cdot 4 \pm 3 \cdot 4$ & $13 \cdot 3 \pm 3 \cdot 7$ & $16 \cdot 8 \pm 7 \cdot 5$ & $0 \cdot 7 \pm 1 \cdot 1$ & $17 \cdot 5 \pm 8 \cdot 1$ \\
\hline 2. $46 \mathrm{M}$ & $8.9 \pm 2.9$ & $17 \cdot 4 \pm 4 \cdot 8$ & $16 \cdot 5 \pm 6 \cdot 7$ & $3 \cdot 2 \pm 2 \cdot 9$ & $19 \cdot 7 \pm 8 \cdot 2$ \\
\hline 3. $46 \mathrm{~F}$ & $8 \cdot 2 \pm 2 \cdot 4$ & $13 \cdot 6 \pm 3 \cdot 1$ & $10 \cdot 6 \pm 2 \cdot 8$ & $5 \cdot 7 \pm 2 \cdot 4$ & $16 \cdot 3 \pm 3 \cdot 1$ \\
\hline 4. $50 \mathrm{~F}$ & $8 \cdot 1 \pm 3 \cdot 5$ & $14 \cdot 4 \pm 4 \cdot 2$ & $22 \cdot 1 \pm 11 \cdot 5$ & $6 \cdot 4 \pm 5 \cdot 0$ & $28 \cdot 5 \pm 12 \cdot 3$ \\
\hline 5. $55 \mathrm{~F}$ & $6 \cdot 2 \pm 2 \cdot 3$ & $11 \cdot 3 \pm 2 \cdot 2$ & $15 \cdot 5 \pm 6 \cdot 2$ & $2 \cdot 7 \pm 1 \cdot 2$ & $18 \cdot 2 \pm 5.9$ \\
\hline 6. $60 \mathrm{~F}$ & $8 \cdot 8 \pm 5 \cdot 0$ & $14 \cdot 1 \pm 6 \cdot 4$ & $20 \cdot 5 \pm 7 \cdot 2$ & $4 \cdot 1 \pm 4 \cdot 1$ & $24 \cdot 5 \pm 7 \cdot 8$ \\
\hline 7. $61 \mathrm{~F}$ & $7 \cdot 8 \pm 2 \cdot 8$ & $12 \cdot 6 \pm 2 \cdot 7$ & $19 \cdot 2 \pm 7 \cdot 7$ & $3 \cdot 0 \pm 2 \cdot 3$ & $22 \cdot 2 \pm 8 \cdot 0$ \\
\hline 8. $75 \mathrm{M}$ & $6 \cdot 4 \pm 2 \cdot 0$ & $12 \cdot 1 \pm 2 \cdot 6$ & $6 \cdot 2 \pm 4 \cdot 1$ & $0.9 \pm 0.7$ & $7 \cdot 1 \pm 4 \cdot 3$ \\
\hline Grand mean $( \pm \mathrm{SD})$ & $7 \cdot 8 \pm 1 \cdot 0$ & $13 \cdot 6 \pm 1 \cdot 9$ & $15 \cdot 9 \pm 5 \cdot 3 \ddagger$ & $3 \cdot 3 \pm 2 \cdot 0 \dagger$ & $19 \cdot 2 \pm 6 \cdot 4 \ddagger$ \\
\hline \multicolumn{6}{|l|}{ Control } \\
\hline 1. $21 \mathrm{~F}$ & $8 \cdot 9 \pm 2 \cdot 4$ & $15 \cdot 4 \pm 3 \cdot 5$ & $10 \cdot 1 \pm 3 \cdot 9$ & $2 \cdot 3 \pm 1 \cdot 1$ & $12 \cdot 4 \pm 3.9$ \\
\hline 2. $31 \mathrm{~F}$ & $8 \cdot 8 \pm 2 \cdot 4$ & $13 \cdot 7 \pm 3 \cdot 5$ & $11 \cdot 8 \pm 5 \cdot 2$ & $1 \cdot 4 \pm 2 \cdot 8$ & $13 \cdot 2 \pm 5 \cdot 0$ \\
\hline 3. $38 \mathrm{~F}$ & $7 \cdot 7 \pm 1 \cdot 8$ & $12 \cdot 9 \pm 3 \cdot 7$ & $6 \cdot 6 \pm 2 \cdot 5$ & $1 \cdot 3 \pm 1 \cdot 5$ & $7 \cdot 9 \pm 2 \cdot 6$ \\
\hline 4. $53 \mathrm{M}$ & $7 \cdot 4 \pm 3 \cdot 8$ & $12 \cdot 3 \pm 4 \cdot 2$ & $3 \cdot 9 \pm 1 \cdot 7$ & $0.6 \pm 0.5$ & $4.5 \pm 1.8$ \\
\hline 5. $54 \mathrm{M}$ & $6 \cdot 3 \pm 2 \cdot 6$ & $11 \cdot 0 \pm 3 \cdot 4$ & $1 \cdot 7 \pm 1 \cdot 2$ & $0 \cdot 3 \pm 0.5$ & $2 \cdot 0 \pm 1 \cdot 2$ \\
\hline 6. $58 \mathrm{~F}$ & $9 \cdot 4 \pm 4 \cdot 3$ & $14 \cdot 9 \pm 5 \cdot 3$ & $11 \cdot 3 \pm 5 \cdot 9$ & $2 \cdot 8 \pm 3 \cdot 3$ & $14 \cdot 1 \pm 8 \cdot 7$ \\
\hline 7. $63 \mathrm{M}$ & $8 \cdot 2 \pm 3 \cdot 6$ & $13 \cdot 8 \pm 5 \cdot 1$ & $6 \cdot 5 \pm 4 \cdot 7$ & $1 \cdot 4 \pm 1 \cdot 3$ & $7 \cdot 9 \pm 5 \cdot 0$ \\
\hline 8. $63 \mathrm{~F}$ & $7 \cdot 3 \pm 3 \cdot 5$ & $12 \cdot 8 \pm 5 \cdot 8$ & $6 \cdot 2 \pm 6 \cdot 9$ & $1 \cdot 6 \pm 1 \cdot 7$ & $7 \cdot 8 \pm 8 \cdot 1$ \\
\hline 9. $68 \mathrm{~F}$ & $7 \cdot 4 \pm 2 \cdot 2$ & $11 \cdot 3 \pm 3 \cdot 2$ & $5 \cdot 5 \pm 3 \cdot 7$ & $0 \cdot 7 \pm 1 \cdot 1$ & $6.2 \pm 3.7$ \\
\hline 10. $75 \mathrm{~F}$ & $7 \cdot 6 \pm 2 \cdot 8$ & $14 \cdot 0 \pm 5 \cdot 1$ & $5 \cdot 4 \pm 3 \cdot 4$ & $1 \cdot 1 \pm 1 \cdot 2$ & $6.5 \pm 3.7$ \\
\hline 11. $80 \mathrm{M}$ & $7 \cdot 2 \pm 1 \cdot 9$ & $14 \cdot 0 \pm 3 \cdot 4$ & $1.9 \pm 1.5$ & $0.7 \pm 0.9$ & $2.6 \pm 1.8$ \\
\hline Grand mean $( \pm \mathrm{SD})$ & $7 \cdot 8 \pm 0.9$ & $13 \cdot 3 \pm 1 \cdot 4$ & $6 \cdot 4 \pm 3 \cdot 4$ & $1 \cdot 3 \pm 0 \cdot 7$ & $7 \cdot 7 \pm 4 \cdot 1$ \\
\hline
\end{tabular}

the pilocarpine-treated eyes the giant vacuoles were larger and more numerous in the endothelium lining the trabecular wall (Fig. 3).

The results obtained in the quantitative investigation of scleral spur displacement are shown in Table 1. There was little overlap of mean values between the 2 groups, and the grand mean of the treated group was about $15^{\circ}$ of arc greater than that of the untreated group (Fig. 4). When Student's $t$ test was applied the mean values were found to be significantly greater in the treated than in the control group $(\mathrm{P}<0.001)$.

The results of the count made of the numbers of giant vacuoles and cell nuclei in the endothelial monolayer surrounding Schlemm's canal are shown in Table 2. As the pilocarpine-treated and the control series were reasonably age-matched, comparison of vacuolar incidence between the 2 groups was considered to be valid. There were well over twice as many giant vacuoles counted in the treated as in the untreated group, and this difference was significant $(P<0.001)$. Overlap between the 2 groups, once again, was minimal (Fig. 5). On the other hand there was no significant difference in the incidence of cell nuclei either in the endothlium of the inner wall or in the endothelium of the whole canal (Fig. 6).

It should be noted that there was considerable variation in the frequency and, to some extent, the 
Fig. 8 Electron micrographs to show the outer meshwork in the untreated eyes of (a) a 68-year-old female, and (b) a 31-year-old female. In the older eye there are accumulations of electron dense material (arrow) in the extracellular space of the endothelial meshwork $(\mathrm{a} \times 1420$; b $\times 1600)$

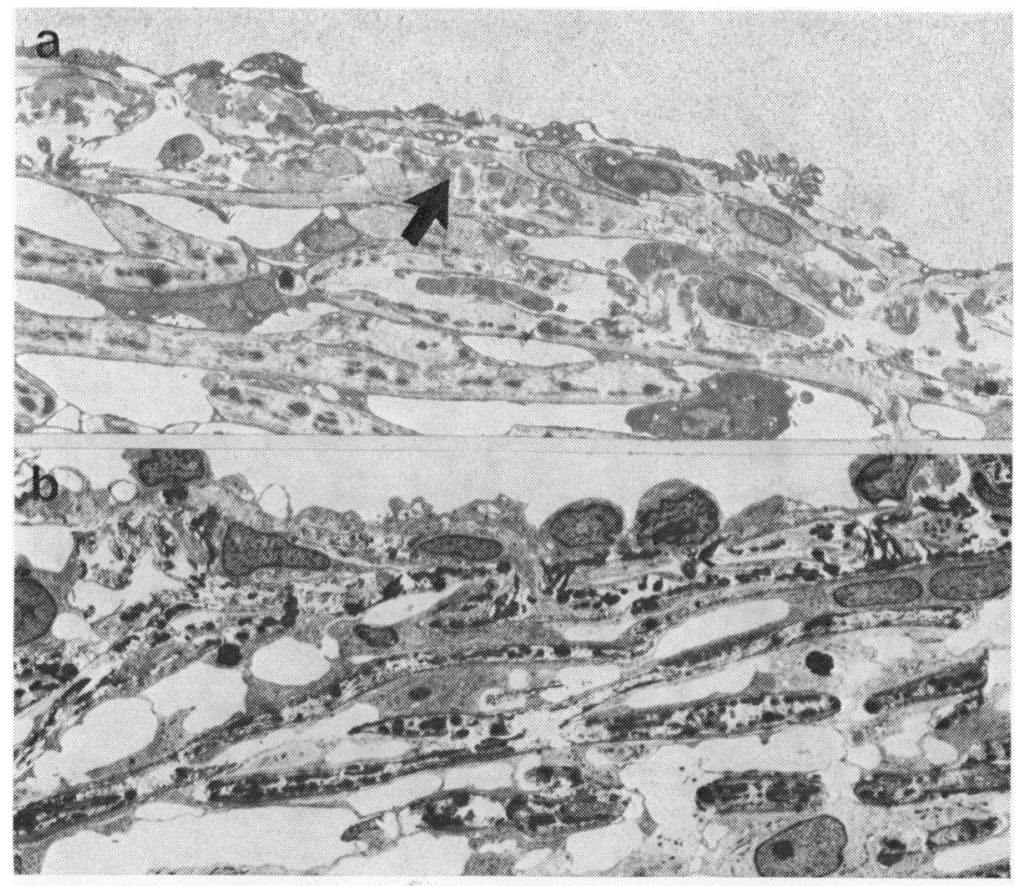

size of the giant vacuoles in the canal endothelium of the control series (Table 2 and Fig. 5). The lowest count was 2.6 giant vacuoles per section and the highest was $14 \cdot 1$ per section, which was more than a fivefold difference in vacuolar incidence. When the counts of giant vacuoles were plotted against the patient's age (Fig. 7), a negative relationship was indicated, but this was not statistically significant $(\mathrm{r}=0 \cdot 58)$.

\section{ELECTRON MICROSCOPY}

There was considerable variation in the appearance of the trabecular wall of Schlemm's canal in the control series, and this to some extent was related to age changes (Rohen and Lütjen-Drecoll, 1971) and probably to a lack of pressure regulation during fixation (Johnstone and Grant, 1973; Grierson and Lee, 1974). One of the most obvious features of the older eye was an increase in the amount of extracellular material in the spaces between the cells of the endothelial meshwork (Fig. 8). The increased density of material in the extracellular spaces, which was assumed to be associated with the ageing process, was due to an accumulation of fine fibrillar material (Fig. 9a) and plaques of electron-dense elastic-like deposits (Fig. 9b). Clumps of $1000 \AA$ $(100 \mathrm{~nm})$ banded substance were present in large amounts in some of the eyes from older individuals. The endothelial meshwork of the pilocarpine-treated eyes was distended to an extent beyond the variation found in the controls, and it was considered that the dilated spaces between the cells were somewhat deficient in extracellular materials (Figs. 10 and 11).

The giant vacuoles of the canal endothelium in both treated and untreated groups were found to be membrane-lined structures which were enveloped by the cytoplasm of a single endothelial cell. Some of the giant vacuoles had pores; pores on the meshwork surface of the vacuoles were larger and more frequent than those on the luminal surface (Figs. 9a, 10, 11, and 12). On occasion, a giant vacuole was shown to be a transcellular channel (Fig. 12).

On the evidence available it appeared that the bulk of the giant vacuoles were blind invaginations from the meshwork surface of the endothelium and that only a small proportion were transcellular channels (Fig. 13). As such, the structures were identical to the endothelial vacuoles which have been described by previous authors and which have been implicated in the transfer of aqueous humour (Holmberg, 1965; Kayes, 1967; Tripathi, 1968, 1972, 1974; Inomata et al., 1972; Grierson and Lee, 1975a). Cellular and extracellular materials were sometimes found within the lumen of the giant vacuoles, and this occurred more frequently in the pilocarpine-treated tissue (Fig. 11).

Sometimes channels were found in the narrow 

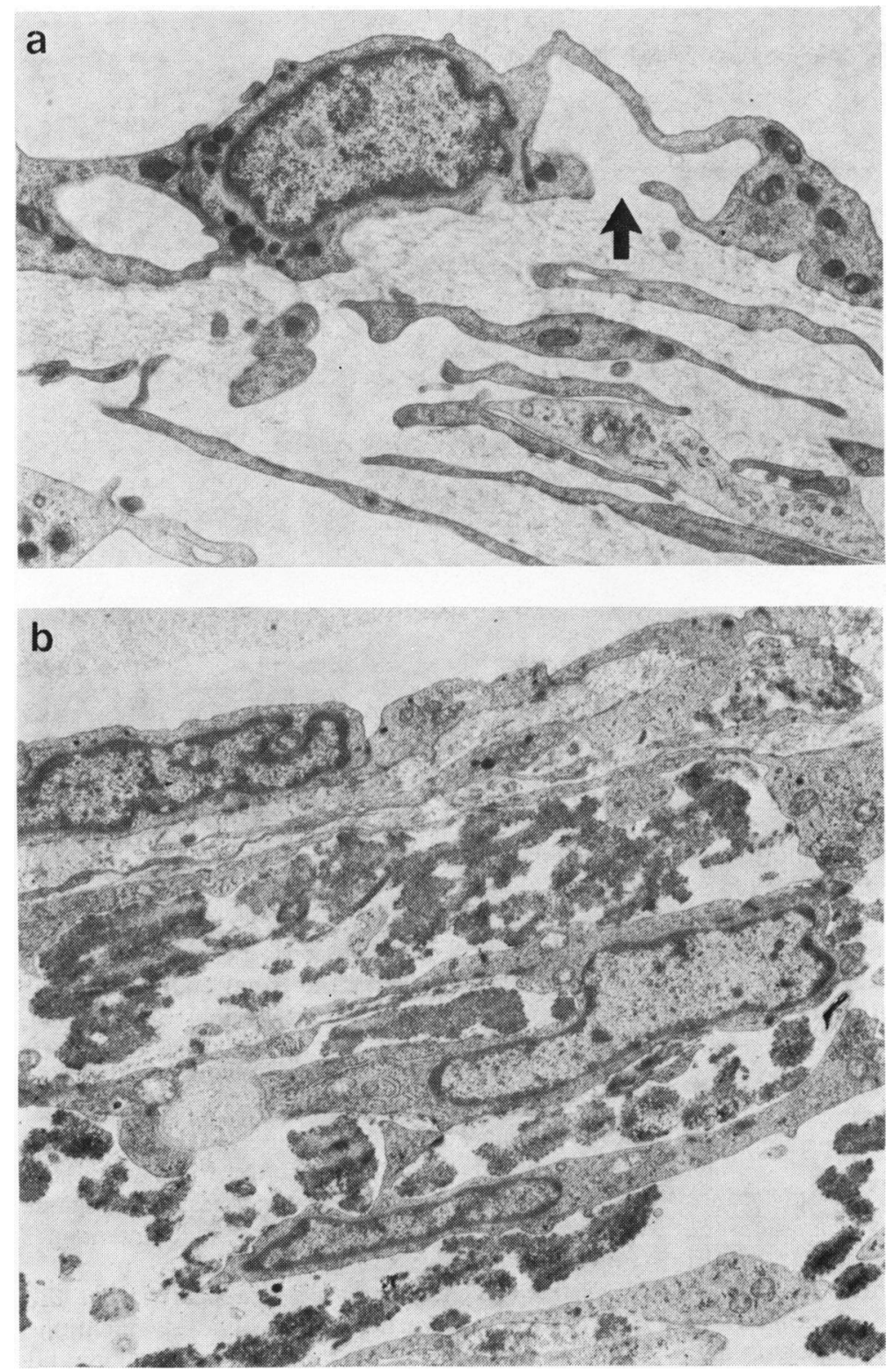

Fig. 9 Electron micrographs to illustrate the 2 types of extracellular material which showed the most marked increase with age. (a) The endothelial meshwork in a 53-year-old male contains fine fibrillar material (a meshwork pore in a giant vacuole is indicated by an arrow). (b) The endothelial meshwork in a 54year-old male contains many electron dense plaques of elastic-like material (a $\times 11800$; b $\times 10100$ ) marginal regions of the endothelial cells (Fig. 13). Occasionally, these non-vacuolar channels contained a wandering cell in transit. The non-vacuolar transcellular channels were found more often in the pilocarpine-treated than in the untreated tissue.

\section{Discussion}

When human tissue is the subject of an investigation of this type there are many features of the experiment which militate against the adoption of a rigid protocol. The different dose levels of the drug, the variable enucleation techniques, and the use of immersion fixation are factors which must introduce variables of indeterminate significance.

Despite the variation found in both the treated and untreated groups the findings confirm the earlier observations by paraffin histology of the effect of pilocarpine on the monkey outflow apparatus (Flocks and Zweng, 1957). This investigation has 
Fig. 10 Electron micrographs to show the outer meshwork in 2 eyes which were treated with pilocarpine. In (a) the outer meshwork is distended, the cells are separated and large electronlucent spaces are prominent.

(b) Shows a cluster of giant vacuoles in which a meshwork pore and a lumen pore are indicated by arrows. (a, 46-yearold male, $\times 1700 ; \mathrm{b}, 60$-year-old female, $\times 4200$ )

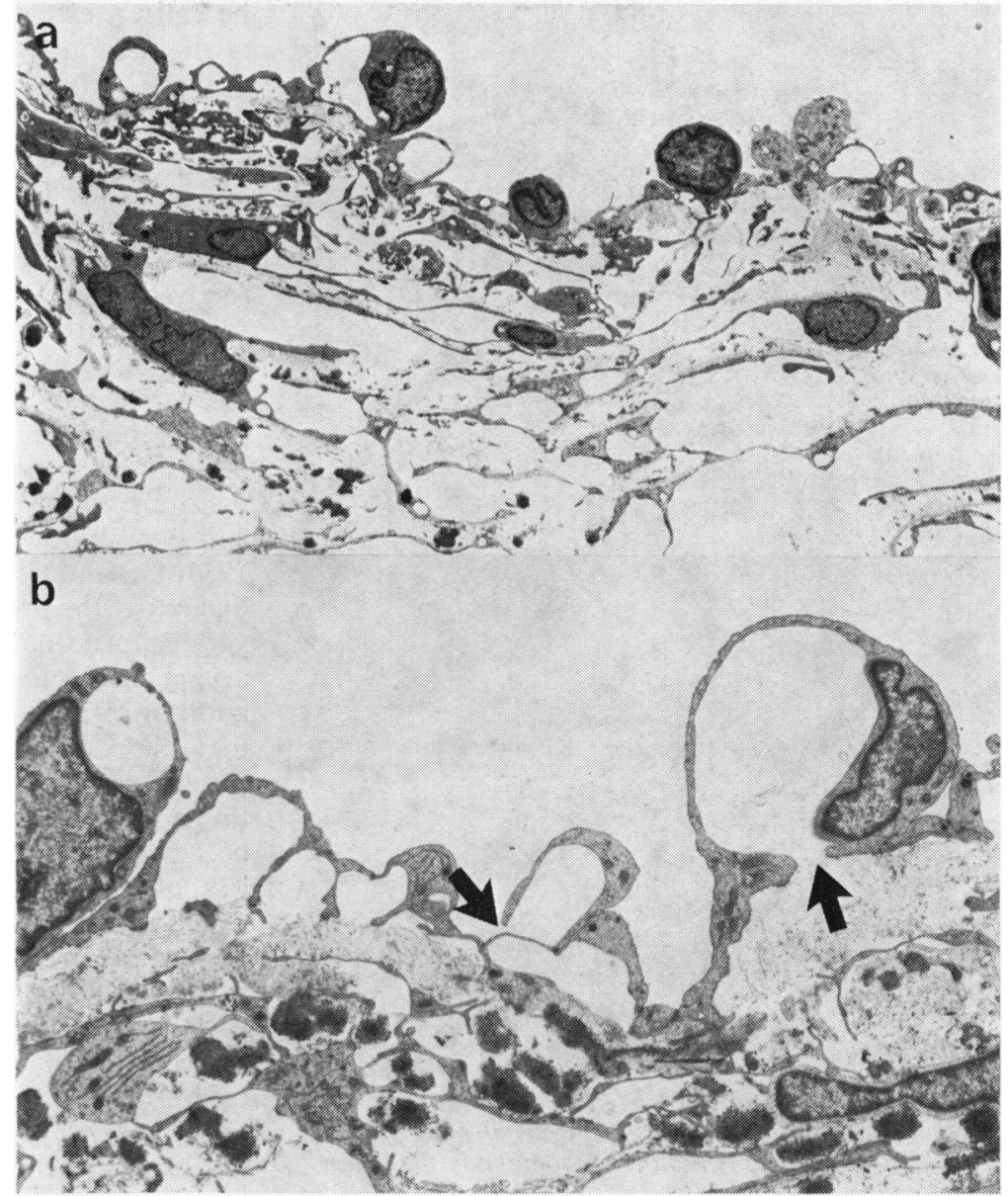

shown that contraction of the ciliary muscle under the action of pilocarpine results in a pull on the scleral spur, so that this fibrous connective tissue projection is pulled backwards and rotated through as much as $15^{\circ}$ of arc. As the corneoscleral trabeculae and the elements of the endothelial meshwork are continuous with the scleral spur, the outer meshwork tissues are both stretched and separated by this lever action (Figs. 14a, b). Thus, the outer meshwork adopts a configuration under the influence of pilocarpine which would decrease the obstruction which is offered to the passage of aqueous humour. As such, the action is similar to the model proposed by Fortin (1931), and using a silicone analogue of the outflow apparatus Allan and Burian (1965) were able to show that component layers in the manner described above resulted in an increased fluid flow across the system.

At this point it must be stressed that the findings in the present investigation were less dramatic than those outlined by Flocks and Zweng (1957). For example, these authors considered that the pathways through the uveal meshwork were enlarged after treatment with pilocarpine. In the present study, however, drug-induced changes in the uveal meshwork were not substantial. An explanation may lie with the action of the primary fixative. The pupil of an eye which has been treated with a miotic tends on fixation to dilate to a greater or lesser extent as a result of uveal neuromuscular blockage. Flocks and Zweng (1957) overcame the problem by killing their experimental animals with systemic administration of pilocarpine at 10 times the lethal dose. Thus the present findings will not indicate the full potential of the miotic on the human outflow system.

The quantitative light microscopic results obtained in this investigation indicated that there was an increase in the number of giant vacuoles in the endothelium of the inner wall of Schlemm's canal in the pilocarpine-treated eyes when compared with 


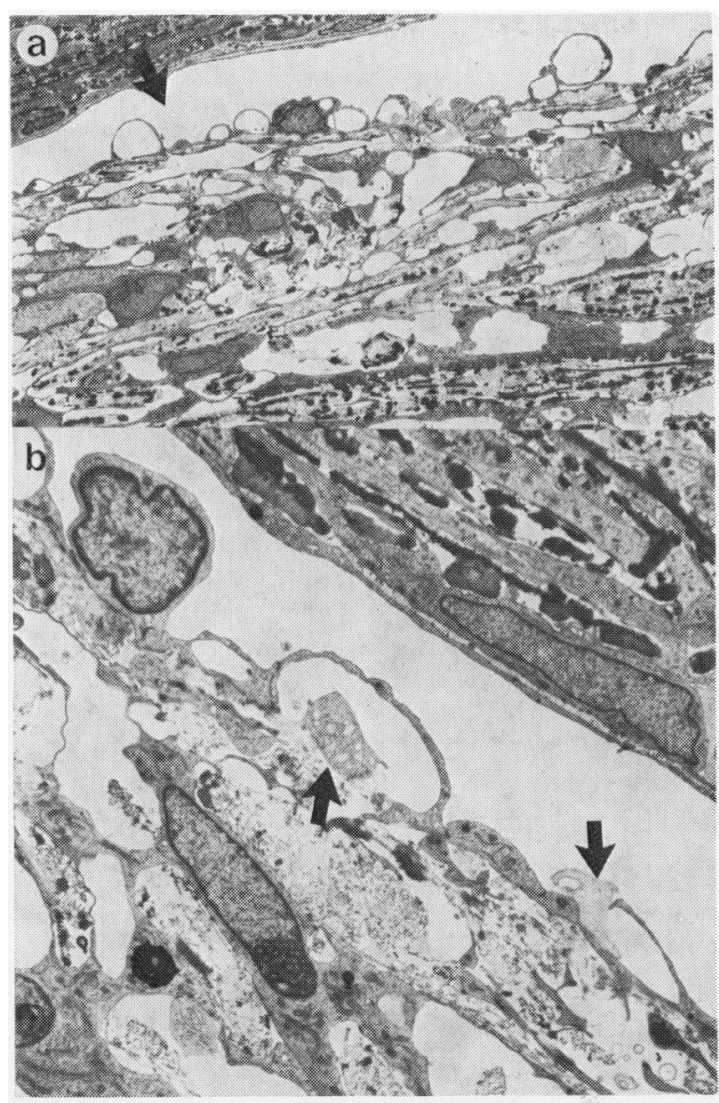

Fig. 11 Electron micrographs to show the outer meshwork in a pilocarpine-treated eye (60-year-old female). In (a) there is prolific vacuolation in the lining endothelium in the inner wall of the canal which is relatively narrowed (arrow). In (b) the giant vacuoles contain cellular and extracellular material within their pores (arrows) $(\mathrm{a} \times 830 ; \mathrm{b} \times 3800)$

the control series. The ultrastructural examination added support to this finding and showed that both vacuolar and non-vacuolar transcellular channels were qualitatively more frequent after pilocarpine treatment, which implies that the potential for aqueous humour transfer is increased.

There was, however, a marked variation between the vacuole counts for the individual eyes in both the treated and the untreated groups. This was emphasised by the remarkably consistent values obtained for the counts of nuclei in which the mean values for the 2 groups were almost identical and the standard deviation was less than $15 \%$. By contrast the variation in the giant vacuole counts for the inner-wall lining endothelium between individuals in the treated and untreated groups was 33 and $53 \%$, respectively. This variation could not be entirely explained by age and could possibly be attributed to the variables previously mentioned, for example, enucleation techniques, fixation techniques, etc. It is also necessary, however, to note the variation in giant vacuole counts from different blocks in the individual eye. In the control group the standard deviation for the inner-wall giant vacuoles varied between 40 and $100 \%$ of the mean (derived from Table 2), while the corresponding calculations for the pilocarpine-treated group showed a 40 to $60 \%$ variation of the SD about the mean. The variation about the mean in the control group could be explained by 2 mechanisms. The first is that in the normal individual outflow system there are selective flow pathways (Rohen, Lütjen, and Bárány, 1967; Rohen, 1969; Grierson and Lee, 1974; Lee and Grierson, 1974). Secondly, it is highly likely that the process of extracellular deposition of various forms of collagen, which occurs as a normal ageing process in the primate outflow system (Rohen and Lütjen-Drecoll, 1971), is of a patchy nature and that this would exaggerate the normal process of focal flow direction. It was therefore of interest that the normal variation in vacuole incidence which occurs between regions in the same eye was much less obvious in the pilocarpine-treated group. It would seem reasonable to conclude that the action of the drug on normal tissue is to extend the drainage properties of the previously confined drainage pathways.

An important corollary in relation to the anatomy of preselected drainage pathways is that distension of the outer layers of the meshwork was associated with the subjective impression that there was a loss of extracellular material. Although an overall increase in tissue volume could explain this impression of extracellular tissue loss, it should be noted that extracellular material was more prominent within the giant vacuoles in the treated group, and the authors are of the opinion that washout of extracellular tissue is a feature of the pilocarpine effect. If this is so, the loss of extracellular material may be an important factor in the increased facility of aqueous outflow which is associated with topical pilocarpine treatment. It is pertinent therefore to describe the observations of Lütjen-Drecoll (1973), who found, in the monkey, Macaca arctoides, that the area of empty spaces beneath the trabecular wall endothelium of Schlemm's canal showed a linear correlation with facility after topical treatment with pilocarpine.

The extracellular tissue of the trabecular meshwork has been shown to contain glycosaminoglycans and glycoproteins (Segawa, 1975; Armaly and Wang, 1975; Grierson and Lee, 1975b) in addition to collagen and elastic-like materials (Rohen and Lütjen- 
Fig. 12 A giant vacuole in a pilocarpine-treated eye (60-yearold female) in which a meshwork pore and a lumen pore (arrows) are identified in a giant vacuole $(\times 6000)$
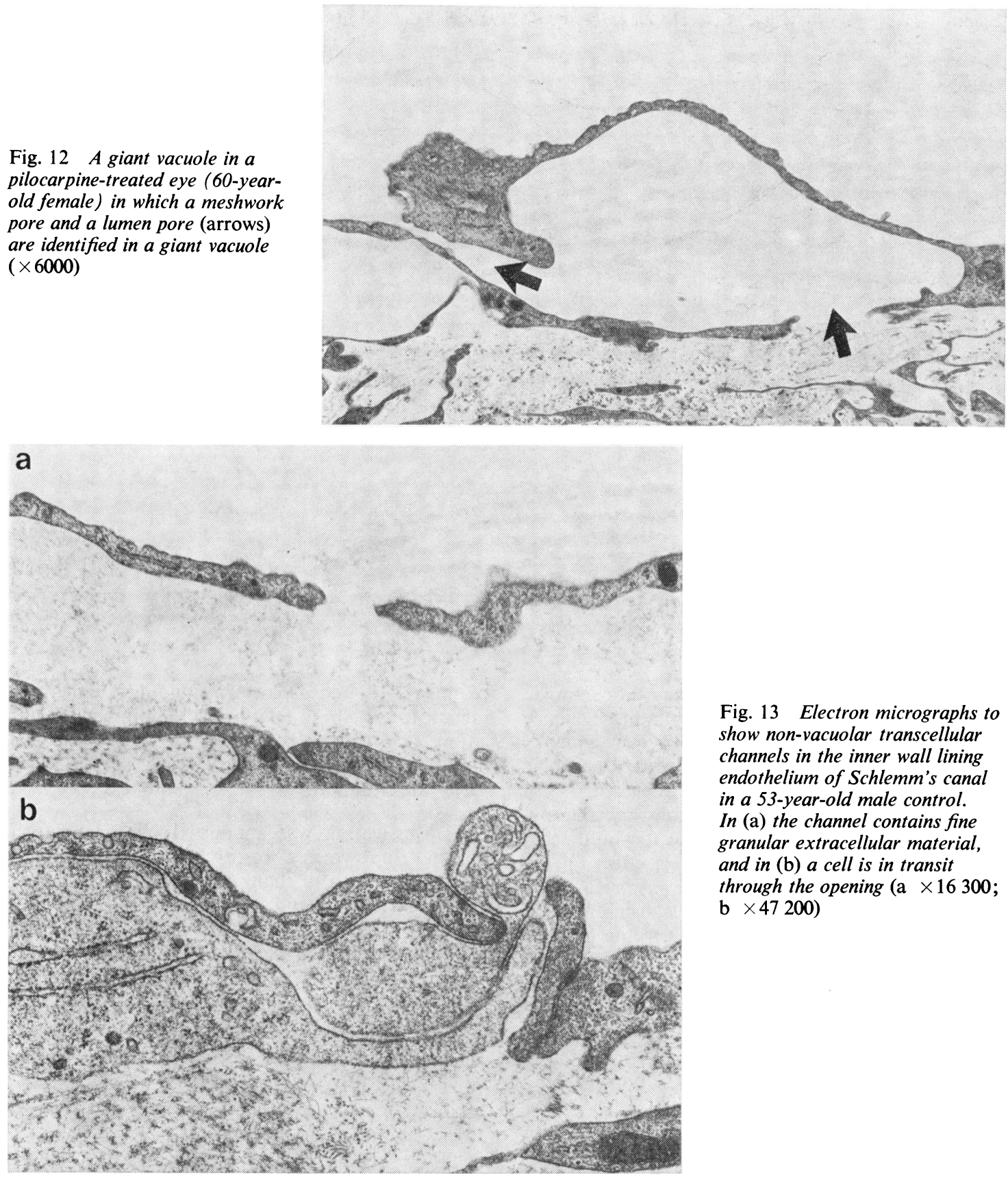

Fig. 13 Electron micrographs to show non-vacuolar transcellular channels in the inner wall lining endothelium of Schlemm's canal in a 53-year-old male control. In (a) the channel contains fine granular extracellular material, and in (b) $a$ cell is in transit through the opening $(\mathrm{a} \times 16300$; b $\times 47200$ )

Drecoll, 1971; Segawa, 1975). Indeed, many reports on the morphology of the outflow system have emphasised the contribution of extracellular tissue in outflow resistance (Inomata et al., 1972; LütjenDrecoll, 1973; Tripathi, 1974; Grierson and Lee, 1975b). While the extracellular tissue will in itself be a factor in resistance, it should be noted that the extracellular materials also are of importance in the maintenance of tissue adhesion. Thus the loss of extracellular tissue by washing out will facilitate the separation of endothelial cells and thus a widening of the extracellular spaces. This situation would be 

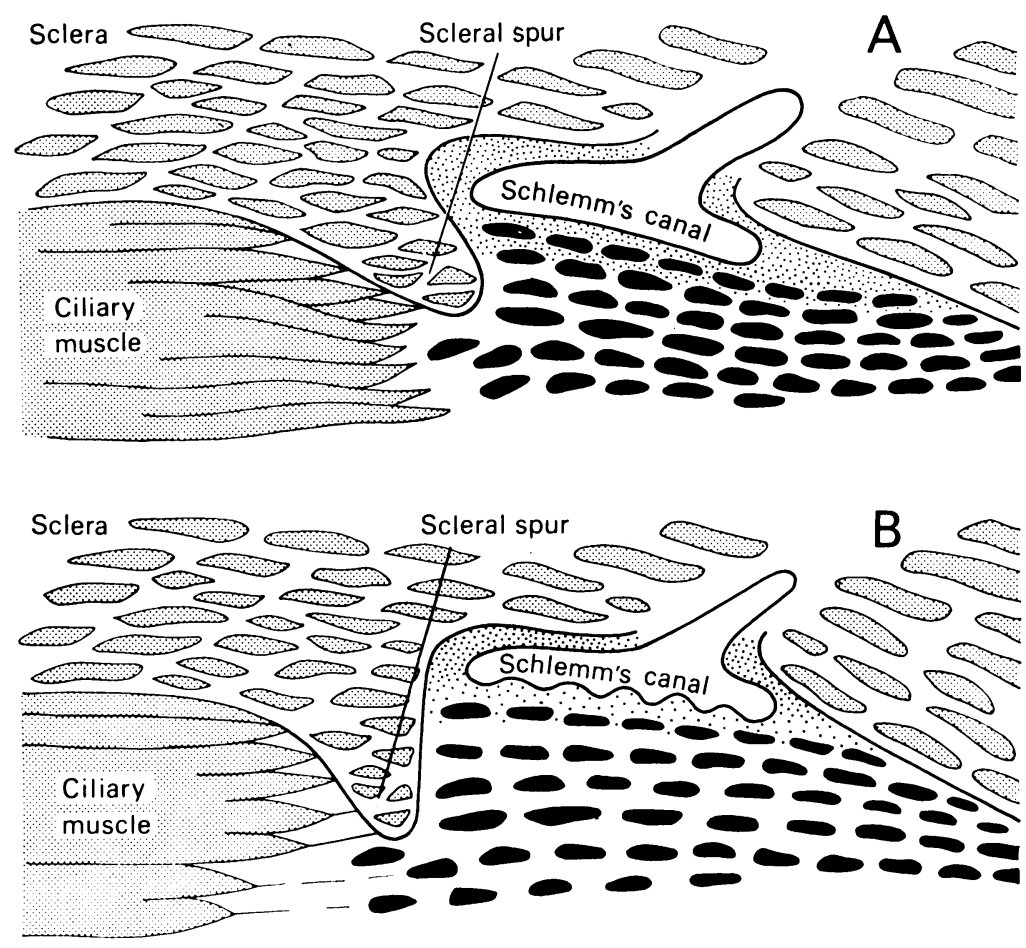

Fig. 14 Diagrams to demonstrate the effects of pilocarpine-induced contraction of the ciliary muscle on the scleral spur and the trabecular tissues. A, untreated; $\mathrm{B}$, treated of particular advantage in primary open-angle glaucoma, in which there is accumulating evidence to suggest that the reduced facility of aqueous outflow is the result of excessive extracellular material (Rohen and Witmer, 1972; Segawa, 1975).

Despite some speculation to the contrary (Bárány, 1966; Holmberg and Bárány, 1966) it would seem unlikely that pilocarpine has a direct effect on the canal endothelium to produce pathways. Kaufman and Bárány (1976) showed that after disinsertion of the ciliary muscle in monkeys pilocarpine did not have a direct action on the outflow apparatus and that the drug action was mediated solely through contraction of the ciliary muscle. In the present investigation the observed increase in the incidence of giant vacuoles was probably due to the increased passage of fluid to the canal endothelium, because the normal obstruction offered by the meshwork tissues was reduced. This is in agreement with previous proposals that giant vacuoles are formed passively and are merely dependent on the amount of aqueous humour which is available for transfer into the canal (Johnstone and Grant, 1973; Grierson and Lee, 1975a).

It is surprising that, in a well-controlled experiment conducted on the outflow system of the vervet monkey, Holmberg and Bárány (1966) found that the frequency of giant vacuoles was halved after treatment with pilocarpine. This finding is somewhat difficult to explain in relation to the present investigation, and further combined morphological and physiological investigations will be necessary to clarify the situation.

This investigation was supported by the Scottish Hospital Endowments Research Trust (Grant 442). The authors wish to thank their clinical colleagues in the west of Scotland for their kind co-operation in the provision of material for this study and Miss Olive M. Rankin for secretarial assistance.

\section{References}

Allan, L., and Burian, H. M. (1965). The valve action of the trabecular meshwork. Studies with silicone models. American Journal of Ophthalmology, 59, 382-389.

Armaly, M. F., and Wang, Y. (1975). Demonstration of acid mucopolysaccharides in the trabecular meshwork of the rhesus monkey. Investigative Ophthalmology, 14, 507-516.

Bárány, E. H. (1966). The mode of action of miotics on outflow resistance. A study of pilocarpine in the vervet monkey Cercopithecus ethiops. Transactions of the Ophthalmological Societies of the United Kingdom, 86, 539-578.

Duke-Elder, S. (1958). System of Ophthalmology, Vol. 7. The Foundations of Ophthalmology, pp. 556-559. Kimpton: London.

Flocks, M., and Zweng, H. C. (1957). Studies on the mode of action of pilocarpine on aqueous outflow. American Journal of Ophthalmology, 44, 380-388.

Fortin, E. P. (1929). Canal de Schlemm y ligamento pectino 
Archives de Oftalmologica, 4, 454-459 (quoted by Allan and Burian).

Gaasterland, D., Küpfer, C., and Ross, K. (1975). Studies of aqueous humor dynamics in man. IV. Effects of pilocarpine upon measurements in young normal volunteers. Investigative Ophthalmology, 14, 848-853.

Grierson, I., and Lee, W. R. (1974). Changes in the monkey outflow apparatus at graded levels of intraocular pressure : a qualitative analysis by light microscopy and scanning electron microscopy. Experimental Eye Research, 19, 21-33.

Grierson, I., and Lee, W. R. (1975a). Pressure induced changes to the ultrastructure of the endothelium lining Schlemm's canal. American Journal of Ophthalmology, 80, 863-884.

Grierson, I., and Lee, W. R. (1975b). Acid mucopolysaccharides in the outflow apparatus. Experimental Eye Research, 21, 417-431.

Grierson, I., and Lee, W. R. (1977). Light microscopic quantitation of the endothelial vacuoles in Schlemm's canal. American Journal of Ophthalmology, 84, 234-246.

Holmberg, A. S. (1965). Schlemm's canal and the trabecular meshwork. An electron microscopic study of the normal structure in man and monkey (Cercopithecus ethiops). Documenta Ophthalmologica, 19, 339-373.

Holmberg, A. S., and Bárány, E. H. (1966). The effect of pilocarpine on the endothelium forming the inner wall of the Schlemm's canal: an electron microscopic study in the monkey Cercopithecus ethiops. Investigative Ophthalmology, 5, 53-58.

Inomata, H., Bill, A., and Smelser, G. K. (1972). Aqueous humor pathways through the trabecular meshwork and into Schlemm's canal in the cynomolgus monkey (Macaca irus). An electron microscopic study. American Journal of Ophthalmology, 73, 760-789.

Johnstone. M. A., and Grant, W. M. (1973). Pressuredependent changes in the structure of the aqueous outflow system of human and monkey eyes. American Journal of Ophthalmology, 75, 365-383.

Kaufman, P. L., and Bárány, E. H. (1976). Loss of acute pilocarpine effect on outflow facility following surgical disinsertion and retrodisplacement of the ciliary muscle from the scleral spur in the cynomolgus monkey. Investigative Ophthalmology, 15, 793-807.

Kayes, J. (1967). Pore structure in the inner wall of Schlemm's canal. Investigative Ophthalmology, 6, 381-394.
Lee, W. R., and Grierson, I. (1974). Relationships between intraocular pressure and the morphology of the outflow apparatus. Transactions of the Ophthalmological Societies of the United Kingdom, 91, 687-705.

Lütjen-Drecoll, E. (1973). Structural factors influencing outflow facility and its changeability under drugs. Investigative Ophthalmology, 12, 280-294.

Mapstone, R. (1977). Normal response to pilocarpine and phenylephrine. British Journal of Ophthalmology, 61 510-511.

Rohen, J. W. (1969). New studies on the functional morphology of the trabecular meshwork and the outflow channels. Transactions of the Ophthalmological Societies in the United Kingdom, 89, 431-447.

Rohen, J. W. Lütjen, E., and Bárány, E. (1967). The relation between the ciliary muscle and the trabecular meshwork and its importance for the effect of miotics on aqueous outflow resistance. Albrecht von Graefes Archiv für klinische und experimentelle Ophthalmologie, 172, 23-47.

Rohen, J. W., and Lütjen-Drecoll, E. (1971). Age changes in the trabecular meshwork in human and monkey eye. In Aging and Development. Pt. 1, pp. 1-36. Akademie der Wissenschaften und der Literatur. Mainz. Schattauer: Stuttgart.

Rohen, J. W., and Witmer, R. (1972). Electron microscopic studies on the trabecular meshwork in glaucoma simplex. Albrecht von Graefes Archiv für klinische und experimentelle Ophthalmology, 175, 143-160.

Segawa, K. (1975). Ultrastructural changes of the trabecular tissues in primary open-angle glaucoma. Japanese Journal of Ophthalmology, 19, 317-338.

Svedbergh, B. (1976). Aspects of the aqueous humor drainage. Functional ultrastructure of Schlemm's canal, the trabecular meshwork and the corneal endothelium at different intraocular pressures. Acta Universitatis Uppsaliensis Abstra. Upps. Diss. Fac. Med., 256, 1-71.

Tripathi, R. C. (1968). Ultrastructure of Schlemm's canal in relation to aqueous outflow. Experimental Eye Research, 7, 335-341.

Tripathi, R. C. (1972). Aqueous outflow pathway in normal and glaucomatous eyes. British Journal of Ophthalmology, 56, 157-174.

Tripathi, R. C. (1974). Comparative physiology and anatomy of the aqueous outflow pathway. In The Eye. Vol 5, pp. 163-356. Edited by H. Davson and L. T. Graham, Jr. Academic Press: London. 